
\title{
R Research Souture \\ Learning dynamics by computational integration of single cell genomic and lineage information
}

\section{Shou-Wen Wang}

Harvard Medical School

Allon Klein ( $\square$ allon_klein@hms.harvard.edu )

Harvard Medical School https://orcid.org/0000-0001-8913-7879

\section{Article}

Keywords: stem cell, cell differentiation, disease onset, drug response, cell dynamics

Posted Date: May 13th, 2021

DOl: https://doi.org/10.21203/rs.3.rs-502709/v1

License: (c) (1) This work is licensed under a Creative Commons Attribution 4.0 International License. Read Full License

Version of Record: A version of this preprint was published at Nature Biotechnology on February 21st, 2022. See the published version at https://doi.org/10.1038/s41587-022-01209-1. 


\section{Learning dynamics by computational integration of single cell genomic and lineage information}

Shou-Wen Wang ${ }^{*, 1}$ and Allon M. Klein ${ }^{*, 1}$

1 Department of Systems Biology, Blavatnik Institute, Harvard Medical School, Boston, MA 02115, USA

*Email: shouwen wang@hms.harvard.edu (S.W.W.); allon klein@hms.harvard.edu (A.M.K.) Abstract

A goal of single cell genome-wide profiling is to reconstruct dynamic transitions during cell differentiation, disease onset, and drug response. Single cell assays have recently been integrated with lineage tracing, a set of methods that identify cells of common ancestry to establish bona fide dynamic relationships between cell states. These integrated methods have revealed unappreciated cell dynamics, but their analysis faces recurrent challenges arising from noisy, dispersed lineage data. Here, we develop coherent, sparse optimization (CoSpar) as a robust computational approach to infer cell dynamics from single-cell genomics integrated with lineage tracing. CoSpar is robust to severe down-sampling and dispersion of lineage data, which enables simpler, lower-cost experimental designs and requires less calibration. In datasets representing hematopoiesis, reprogramming, and directed differentiation, CoSpar identifies fate biases not previously detected, predicting transcription factors and receptors implicated in fate choice. Documentation and detailed examples for common experimental designs are available at https://cospar.readthedocs.io/.

\section{Introduction}

In tissue development, regeneration, and disease, cells differentiate into distinct, reproducible phenotypes. A ubiquitous challenge in studying these processes is to order events occurring during differentiation ${ }^{1-3}$, and to identify events that drive cells towards one phenotype or another. This challenge is common to understanding mechanisms in embryo development, stem cell self-renewal, cancer cell drug resistance, and tissue metaplasia ${ }^{1-3}$.

At least two observational strategies help to order cellular events. Single-cell genome-wide profiling - such as by single-cell RNA sequencing (scRNA-seq) - offers a universal and scalable approach to observing dynamic states by densely sampling cells at different stages $^{3-10}$. However, scRNA-seq alone does not identify which early differences between cells drive or correlate with fate ${ }^{2,11-13}$. Conversely, lineage tracing offers a complementary family of methods that can clarify long-term dynamic relationships across multiple cell cycles. To carry out lineage tracing, individual cells are labeled at an early time point ${ }^{1-3}$. The state of their clonal progeny is analyzed at one or more later time points (Fig. 1a). 
Recently, a number of efforts from us and others have integrated lineage-tracing with single-cell genome-wide profiling (hereafter LT-scSeq) using unique, heritable, and expressed DNA barcodes ${ }^{2,13-21}$. These technologies identify cells that share a common ancestor and define their genomic state in an unbiased manner. LT-scSeq experiments have been used to successfully identify when fate decisions occur ${ }^{13,14}$, novel markers for stem cells ${ }^{16}$, and pathways which control cell fate choice ${ }^{14,16}$. The simplest of these methods labels cells at one time point ${ }^{13}$ (Fig. 1b); more complex methods allow the accumulation of barcodes over successive cell divisions to reveal the substructure of clones $^{2,13-21}$ (Fig. 1c).

Emerging LT-scSeq methods have been successful at revealing novel regulators of cell fate $^{14,16}$ and the fate potential of early progenitors ${ }^{13,14}$, but they also present challenges that may limit their utility in practice. We identified at least five technical and biological challenges that affect experimental design and interpretation (Fig. 1f). These include stochastic

differentiation and variable expansion of clones $^{22}$ (Fig. 1f-i), cell loss during analysis (Fig. 1f-ii), barcode homoplasy wherein cells acquire the same barcode despite not having a lineage relationship ${ }^{2}$ (Fig. 1f-iii), access to clones only at a single time point ${ }^{23,24}$ (Fig. 1f-iv), and clonal dispersion due to a lag time between labeling cells and the first sampling (Fig. 1f-v). Addressing these problems should greatly simplify the design and interpretation of LT-scSeq assays and put them in the hands of a wider research community. To our knowledge, there is not yet an analysis method that systematically overcomes these problems.

Here, we develop a robust and generalizable computational approach to analyze LT-scSeq experiments. We begin with a model of clonal dynamics in which cells divide, differentiate, or are lost from the sampled tissue in a stochastic manner, with rates that are state-dependent (Supplementary Fig. 1a). We use this model to learn from the data the fraction of progeny of cells, initially in one state, which are found to occupy a second state after some time interval (Fig. 1d, Supplementary Fig. 1b,c). Our approach captures differentiation bias and fate hierarchies, and can reveal genes whose early expression is predictive of future fate choice.

\section{Results}

\section{Dynamic inference from clonal data with state information.}

A formalization of dynamic inference is to identify a transition map, a matrix $T_{i j}\left(t_{1}, t_{2}\right)^{7,25}$. We define $T_{i j}\left(t_{1}, t_{2}\right)$ specifically as the fraction of progeny of a cell, initially in some state $i$ at time $t_{1}$, that occupy state $j$ at time $t_{2}$ (Fig. 1d, Supplementary Fig. 1c). This transition matrix represents a coarse-grained view of the cell dynamics : it already combines the effects of cell division, loss, and differentiation (Supplementary Fig. 1d). As will be seen, even learning $T_{i j}\left(t_{1}, t_{2}\right)$ will prove useful for several applications (Fig. 1d).

We make two reasonable assumptions about the nature of biological dynamics to constrain inference of the transition map. We assume the map to be a sparse matrix, since most cells 
can access just a few states during an experiment (Fig. 1e, left panel). And we assume the map to be locally coherent, meaning that cells in similar states should share similar fate outcomes (Fig. 1e, right panel). These constraints together force transition maps to be parsimonious and smooth, which makes them robust to practical sources of noise in LT-scSeq experiments (Supplementary Fig. 1e). Box 1 formalizes the two constraints and lays out the technical foundation for inferring a transition map by coherent sparse (CoSpar) optimization (see schema in Fig. 2a; Supplementary Fig. 2). As inputs, CoSpar requires a clone-by-cell matrix $I(t)$ that encodes the clonal information at time $t$, and a data matrix for observed cell states (e.g. from scRNA-seq).

CoSpar is formulated assuming that we have information on the same clones at more than one time point. More often, one might observe clones at only one time point $t_{2}$. For these cases CoSpar jointly optimizes the transition map $T$ and the initial clonal data $I\left(t_{1}\right)$ (Fig. 2b; Methods). In this joint optimization, one must initialize the transition map; we have shown that the final result is robust to initialization (Supplementary Fig. 3e; Supplementary Fig. 4c,d). This approach can be used for clones with nested structure (Supplementary Fig. 4f-h). Finally, coherence and sparsity provide reasonable constraints to the common problem of predicting dynamics from state heterogeneity alone without lineage data ${ }^{7}$. We extended CoSpar to this case. Thus, CoSpar is flexible to different experimental designs, as summarized in Fig. 1 d.

\section{Box 1: Coherent Sparse Optimization}

In a model of stochastic differentiation, cells in a clone are distributed across states with a time-dependent density profile $P(t)$. A transition map $T$ directly links clonal density profiles $P\left(t_{1,2}\right)$ between time points:

$$
P_{i}\left(t_{2}\right)=\sum_{j} P_{j}\left(t_{1}\right) T_{j i}\left(t_{1}, t_{2}\right)
$$

From multiple clonal observations, our goal is to learn $T$. To do so, we denote $I(t)$ as a clone-by-cell matrix and introduce $S$ as a matrix of cell-cell similarity over all observed cell states, including those lacking clonal information. The density profiles of all observed clones are estimated as $P(t) \approx I(t) S(t)$.

With enough clonal information, $T\left(t_{1}, t_{2}\right)$ could in principle be learnt by matrix inversion. However, the number of clones will always be far less than the number of states. To constrain the map, we require that: 1) $T$ is a sparse matrix (Fig. 1e, left panel); 2) $T$ is locally coherent (Fig. 1e, right panel); and 3) $T$ is a non-negative matrix. With these requirements, the inference can be formulated as the following optimization problem:

$$
\min _{T} \underbrace{\|T\|_{1}}_{\text {Sparsity }}+\underbrace{\alpha\|L T\|_{2}}_{\text {Coherence }}, \quad \text { s.t. } \underbrace{\left\|\mathbf{P}\left(t_{2}\right)-\mathbf{P}\left(t_{1}\right) T\left(t_{1}, t_{2}\right)\right\|_{2} \leq \epsilon}_{\text {Clonal dynamics }} ; T \geq 0 ; \text { Normalization }
$$


$\|T\|_{1}$ quantifies the sparsity of the matrix $T$ through its L1 norm, and $\|L T\|_{2}$ quantifies the local coherence of $T$ ( $L$ being the Graph Laplacian of the cell state similarity graph, and $L T$ being the local divergence). The remaining constraints enforce the observed clonal dynamics, non-negativity of $T$, and map normalization, respectively. At $\alpha=0$, the minimization takes the form of $\mathrm{Lasso}^{26}$, an algorithm for compressed sensing. Our formulation extends compressed sensing from vectors to matrices, and to enforce local coherence. The local coherence extension is reminiscent of the fused Lasso problem ${ }^{27}$. An iterative, heuristic approach solves the CoSpar optimization efficiently (Fig. 2a; Supplementary Fig. 2). See Methods and Supplementary Notes 1-3 for further details.

Computer simulations validate that CoSpar recovers dynamics with quantitative accuracy, and they establish that CoSpar inference is robust to two errors typical of LT-scSeq -barcode homoplasy and clonal dispersion. We modeled cells progressing through a sequence of gene expression states either towards a single fate (Fig. 3a) or bifurcating into two fates (Fig. 3e), with clones sampled in a manner representative of LT-scSeq experiments ${ }^{13,14}$. With 1000 clones - typical of real experiments - mean transition rates inferred by CoSpar were within 3 standard deviations of the actual transition rate $98 \%$ of the time (TPR $>98 \%$, Fig. $3 d$ ) and the distribution of progeny fates showed $85 \%$ Pearson correlation to ground truth (Fig. 3j). Inferences remained similarly accurate with as few as 30 clones (Fig. 3d). CoSpar was robust to barcode homoplasy, and only detectably lost accuracy when all lineage barcodes mixed more than ten clones on average (Fig. 3a-d). This degree of homoplasy is far higher than expected in most experiments. Further, CoSpar was robust to clonal dispersion, simulated by sampling clones at increasing times post-barcoding (Fig. 3f-i). Conversely, approaches used in previous work, which average the transitions between cells observed in each clone at different time points ${ }^{13}$, are severely affected by both lag time and barcode homoplasy (Fig. 3d,g,i).

\section{CoSpar predicts early fate bias in hematopoiesis.}

We applied CoSpar to published datasets from three independent experiments. The first experiment tracked hematopoietic progenitor cells (HPCs) differentiating in culture, with clones sampled on days 2, 4 and 6 post-barcoding (Fig. $4 \mathbf{a}, \mathbf{b})^{13}$. During this time, cells progressed from a heterogeneous pool of HPC states into ten identifiable differentiated cell types. We used all clonal data to generate a ground truth for the early fate bias towards either the monocyte or neutrophil fate, using the method from Weinreb et.al. ${ }^{13}$ (Fig. 4c).

As a baseline for comparison, we applied CoSpar to predict HPC fate bias using state information alone (Fig. 4e). For this and further comparisons, we report the accuracy of fate prediction using Pearson correlation of predicted fate bias with that observed using all clonal data ('ground truth'). Even without access to any clonal data, CoSpar could resolve early fate bias at a performance close to the upper bound defined by cross-validation of the ground-truth data (CoSpar correlation $\mathrm{R}=0.69$; ground-truth $\mathrm{R}=0.72$ ) (Fig. 4e,g; 
Supplementary Fig. 6a). This performance reflects improvements from enforcing coherence and sparsity ( $R=0.51-0.54$ prior to CoSpar; Fig. 4d; Supplementary Fig. 3f). However, the prediction based on state information alone is limited because it is sensitive to the choice of distance metric used in analysis (Fig. 4g; Supplementary Fig. 3e).

Clonal information eliminated the sensitivity to distance metric. To show this, we applied CoSpar to data restricted in time, or restricted in its quality and depth. Using even a single time point of clonal data (day 6), CoSpar recovered early fate bias (Fig. 4 f; $R=0.68$ ), and it did so robustly over a range of parameters and choices of distance metrics (Fig. $4 \mathbf{g}$;

Supplementary Fig. 3e). Further, it recovered the differentiation hierarchy seen in the correlation of clonal barcodes across all cell types (Supplementary Fig. 3c,d). When using a sub-sampled dataset from the top 15\% most dispersed clones as ranked by day 4 intra-clone distance (Fig. 4b), CoSpar performed similarly well, and outperformed the method from Weinreb et al., which was used to analyze this data originally ${ }^{13}$ (Fig. $4 \mathbf{h}$,i; Supplementary Fig. 3a,b). Thus, CoSpar successfully facilitates analysis of clones at a single time point, or using a fraction of the original data collected in this example.

These benchmarks suggest that CoSpar should be able to predict fate biases not previously recognized. We investigated fate biases in the Gata $1^{+}$states that give rise to five mature fates: megakaryocyte (Mk), erythrocyte (Er), mast cell (Ma), basophil (Ba), and eosinophil (Eos) (Fig. $4 \mathbf{a}, \mathbf{k})$. In culture, $\mathrm{Mk}$ and $\mathrm{Er}$ arise from a common progenitor (MEP), and $\mathrm{Ba}$, Eos and $\mathrm{Ma}$ are produced by a different progenitor (BEMP) ${ }^{30,31}$. Existing studies of these progenitors are hampered by the lack of good markers. While molecular signatures of FACS-sorted MEP have been explored recently ${ }^{32}$, less is known about the transcriptomic identity of BEMPs. This dataset provides an opportunity to predict the molecular identity of these early progenitors. The original method used to analyze this data finds very few genes distinguishing BEMPs and MEPs (Supplementary Fig. 3g-i). Applying CoSpar, we predict an early fate decision boundary between MEP and BEMPs (Fig. 4j,k), which correlates with the early expression of genes later associated with the resulting cell types (S/c14a1 for $\mathrm{Mk}^{32}$, Thy 1 for $\mathrm{Ba}^{33}$; Fig. 4I), and with the transcription factor (TF) Cebpa that regulates Eos and Ba differentiation ${ }^{30}$. We identified 377 known and novel putative fate-associated genes (Fig. 4m; Supplementary Table 1). Differences between the putative BEMPs and MEPs are evident in scRNA-seq data, and clonal data integrated by CoSpar supports that the differences are associated with functional fate bias. This analysis highlights that CoSpar can identify fate-predictive genes from limited LT-scSeq data.

\section{CoSpar reveals early fate bias in reprogramming.}

The second experiment we analyzed tracked cells during the reprogramming of fibroblast cells over 28 days into endodermal progenitors (Fig. 5a) ${ }^{14}$. In this experiment, approximately $30 \%$ of cells successfully reprogrammed; the remainder failed. Clonal analysis with cumulative barcoding was used to identify these cells early and predicted features that regulate their fate (Fig. 5b,c). We used clones strongly enriched in one of the two fates, identified by the original 
study, to generate the ground truth for early fate bias, and we then used it to benchmark CoSpar.

To evaluate CoSpar, we revisited this experiment after discarding over $90 \%$ of clones, and we specifically retained clones that show the least bias in reprogramming outcomes. Despite deliberately using down-sampled low-quality data, CoSpar recapitulated fate bias: the predicted progenitors of reprogrammed and failed cells share 73 out of 100 marker genes with the ground truth population (Fig. 5f), including genes previously showing strong positive and negative association with reprogramming success (Apoa1, Spint2, Col1a2, Peg3), as well as Mettl7a1, which was found to improve reprogramming ${ }^{14}$. These genes could be associated with fate bias using as few as ten clones, even when deliberately selecting clones with minimal fate bias (Fig. 5d,e; Supplementary Fig. 4b). By contrast, the analytical approach used in the original study ${ }^{14}$ failed to identify fate-predictive gene expression after such severe reduction in data quality (Fig. 5e,f; Supplementary Fig. 4b). Further, CoSpar performed robustly when using only clonal data from the final time point of the experiment (Fig. $\mathbf{5 g}, \mathbf{h}$; Supplementary Fig. 4c-e).

As in hematopoiesis, it is instructive to see the information encoded in clonal relationships. When applying CoSpar without clonal data, we found that CoSpar could predict the same early fate biases (Fig. $5 \mathbf{g}$, Middle panel), but is again sensitive to the distance metric used (Fig. 5h). A different distance metric performs best here from the hematopoiesis dataset, suggesting that there is no simple 'best-practice' approach to dynamic inference in the absence of clonal data.

Finally, we applied CoSpar to predict fate bias at the earliest available time point after reprogramming is initiated (day 3 ), where no clonal information is available and fate bias remains unexplored ${ }^{14}$. Using clonal information, CoSpar predicts strong fate biases (Fig. 5i), arguing that future reprogramming success is established very early on. This prediction is supported by the differential expression of transgene FoxA1-HNF4a (a TF cocktail to induce reprogramming), the reprogramming marker gene Apoa1, and failed trajectory marker Col1a2 and $D I k 1^{14}$ (Fig. $5 \mathrm{j}$ ). We also identified multiple genes predicted to correlate with fate bias on day 3 and whose significance in reprogramming has not been previously established (Fig. 5k; Supplementary Table 2).

\section{CoSpar predicts early fate bias during lung directed differentiation.}

In the third experiment, human pluripotent stem cells were differentiated into distal lung alveolar epithelial cells (induced alveolar epithelial type 2 cells, or iAEC2s) ${ }^{23,34}$. Here, clonal and transcriptomic information were profiled jointly on day 27 after initial barcoding on day 17, and a separate time-course experiment produced scRNA-seq data for 6 time points, including days 17 and 21(Fig. 6a). In this study, Hurley et al. reported the existence of clones derived from multipotent cells on day 17 but did not investigate their fate biases. A re-examination of the clonal data, however, suggests strong fate biases as early as day 17 . Out of the 272 
clones, $25 \%$ were enriched in either the iAEC2 or non-iAEC2 clusters (FDR=0.01), and clonal compositions differed significantly from that of randomized clones (Fig. 6b). Accordingly, clonal representation of iAEC2s anti-correlates with other fates (Supplementary Fig. 5b,c). We investigated signatures that could predict effectors of fate bias among day 17 progenitors.

Applying CoSpar, we assigned a putative fate bias to each of the cells seen on day 17 . CoSpar predicts some cells to be strongly biased in cell fate (Fig. 6c), and also the existence of unbiased multipotent states; these strongly overlap with highly proliferating cell states on day 17 and are consistent with large clones hosting multiple endodermal lineages on day 27 (Supplementary Fig. 5d). As a control, we expected weaker fate biases earlier in differentiation, which is confirmed by applying CoSpar to cells two days earlier (day 15, Supplementary Fig. 5e-g). Among genes differentially expressed between the two biased populations on day 17, we identified several established TFs that regulate lung differentiation:

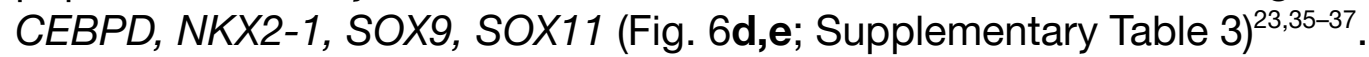

\section{Discussion}

Here we have developed a computational framework for systematically inferring dynamic transitions by integrating state and clonal information. It extends the problem of compressed sensing. Our method takes advantage of reasonable assumptions on the nature of biological dynamics: that cells in similar states behave comparably, and that cells limit their possible dynamics to give sparse transitions. Using published datasets, we demonstrated that coherent sparse optimization relates molecular heterogeneity of cells to their future fate outcomes in a manner that is robust to typical sources of experimental error (Fig. 1f), using as little as $5-10 \%$ data originally collected in prior experiments. The computational methods used in each original study to analyze clonal data were sensitive to clonal dispersion and to down-sampling of data. CoSpar also successfully predicted early fate biases in these datasets using only clonal information from the last time point. When clonal data was removed entirely, results were sensitive to the choice of distance metric, and no single approach optimally inferred fate bias across all data sets.

The robustness of CoSpar could greatly simplify the design of LT-scSeq experiments, by enabling experiments with fewer cells, fewer clones, or fewer time points. In all three datasets considered here, CoSpar reveals clear early fate boundaries that were not previously reported, yet in agreement with the heterogeneity of key transcription factors and fate determinants. We predicted novel transcription factors and markers in each case, and they could facilitate enriching and manipulating the desired fate outcomes.

The examples we have analyzed specifically relate to LT-scSeq implemented using LARRY $Y^{13,23}$ and CellTagging ${ }^{14}$, but CoSpar is not limited to these technologies. The state measurement can be transcriptomic (via scRNA-seq or RNA fluorescence in situ hybridization (FISH) ${ }^{38}$ ), as shown above, as well as proteomic and epigenomic; and lineage tracing can be achieved with 
static DNA barcodes ${ }^{13,23}$, endogenous mutations ${ }^{39}$, or exogenous DNA constructs that accumulate mutations over time, like CRISPR-based editing ${ }^{2,17,18,40,41}$. CoSpar can thus facilitate interpretation of the rapidly evolving field of LT-scSeq, and thus accelerate exploration of development and disease.

CoSpar also has limitations, which directly follow from its central assumption. By enforcing coherent fate choices between similar cells (Fig. 2a), CoSpar becomes sensitive to choices in measuring cell-cell similarity, and to the degree of smoothing used in implementing the algorithm (Supplementary Fig. 2c). Thus, CoSpar will fail to identify fate biases when heterogeneity relevant to cell fate is not measured, or when it is filtered out during data analysis, or due to over- or under-smoothing. In addition, when inferring progenitor bias from clones observed at a single late time point, CoSpar necessarily leans more strongly on state information, and it might fail when heterogeneity in the later population cannot be related to heterogeneity in the initial population. Despite these caveats, CoSpar provided sensible predictions in the cases examined here.

Coherent sparse optimization could prove useful for applications beyond dynamic inference. Several problems require learning locally coherent maps from few and noisy measurements. Such problems occur, for example, when integrating two sets of measurements in the same system ${ }^{42,43}$ (batch correction and multi-omics), decoding spatial transcriptomes from composite FISH measurements ${ }^{44}$, and inferring responses of a system to individual perturbations from composite perturbation readouts ${ }^{45-47}$. Outside of biology, the association of measurements in one modality with sparse measurements in another can occur in marketing and social networks ${ }^{48}$. Forcing coherence and sparsity constraints could greatly improve map inference in general, reducing the cost of data acquisition and enabling new discoveries. 


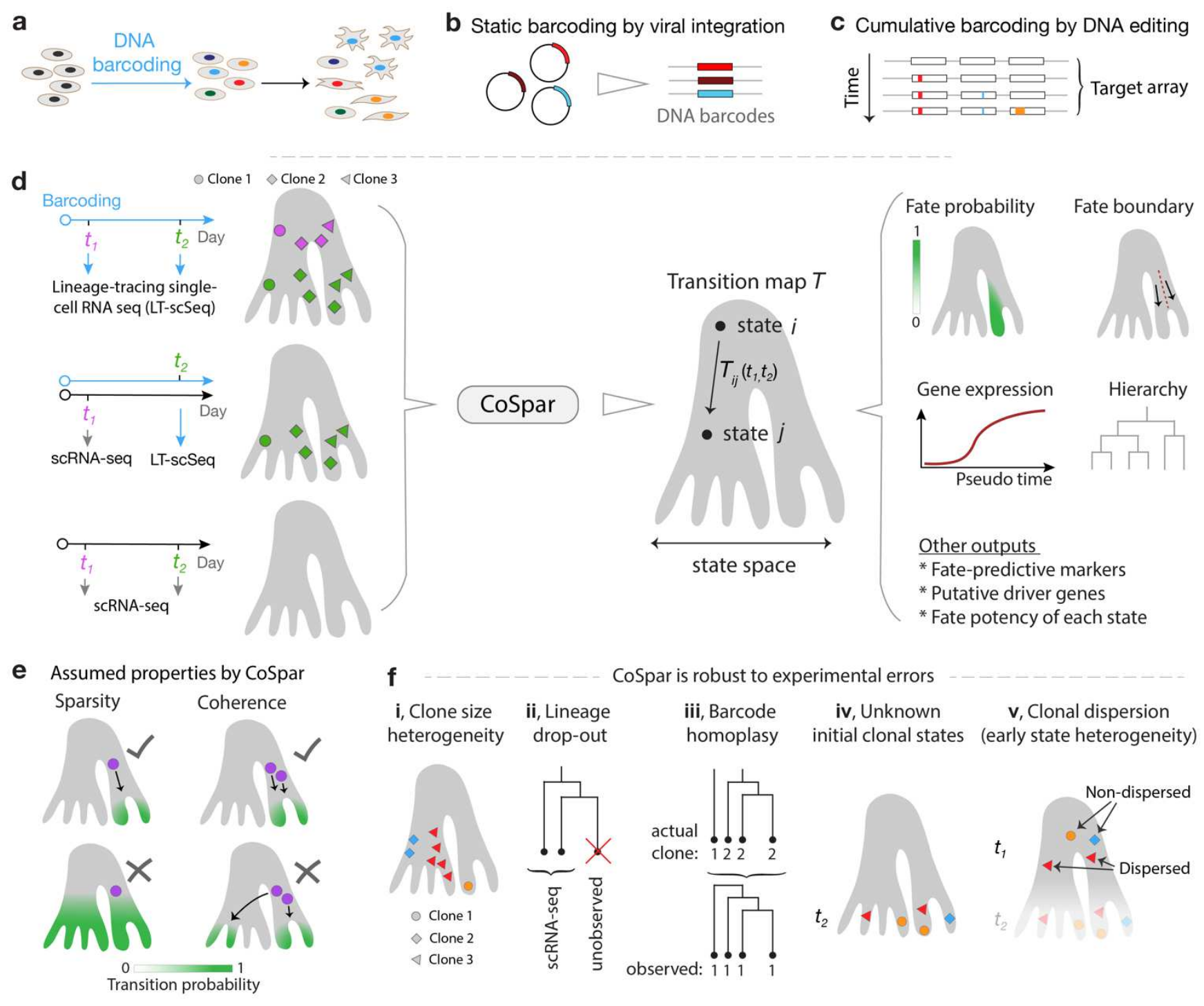

Fig. 1. Integrative analysis of lineage tracing and transcriptome data. a, Lineage-tracing single cell genomics (LT-scSeq) experiments simultaneously measure cell phenotypes and clonal lineage (indicated by colors). b-c, LT-scSeq assays encode lineage information with static DNA barcodes or cumulative barcoding. $\mathbf{d}$, CoSpar unifies analysis of different experimental designs to infer transition maps (see text) to reveal fate boundaries, lineage hierarchy, putative markers, and putative fate-determinants. Here and below, the shaded gray regions schematically show a manifold of observed single cell genomic states. e, Two key assumptions constrain dynamic inference by CoSpar. f, Stereotypical challenges in clonal analysis. Single labeled cells can give rise to clones with a wide dispersion in size; LT-scSeq loses cells during analysis leading to loss of clonal structure; barcode homoplasy occurs when cells from different clones present the same barcode due to experimental limitations; progenitor states are not observed when clones are only observed upon tissue dissociation; clonal dispersion occurs when early clonal states are heterogeneous due to the lag time between barcoding and profiling. 
a

Coherent sparse optimization (CoSpar) for re-sampled clonal data

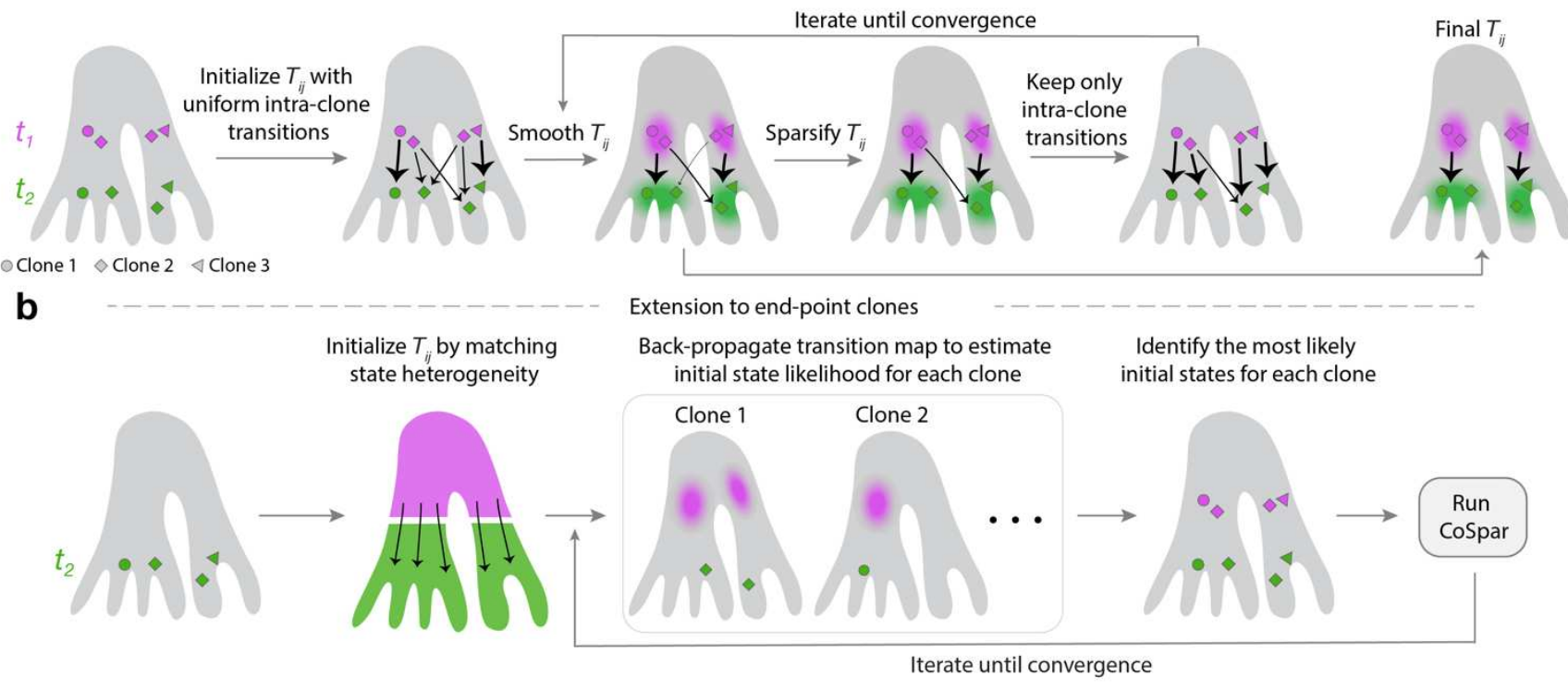

Fig. 2. The CoSpar algorithm. a, When clones are resampled at two time points, a transition map is inferred by iteratively enforcing observed clonal transitions, coherence (by smoothing) and sparsity until convergence is achieved. (See details and derivation in Methods and Supplemental Note 3). b, When clones are observed only once, we infer their progenitor fate bias and identity by first initializing a transition map without clonal information, then iteratively (1) back-propagating the map to predict clonal progenitor identity and (2) learning the transition map as in a until the map and progenitor identities jointly converge. 

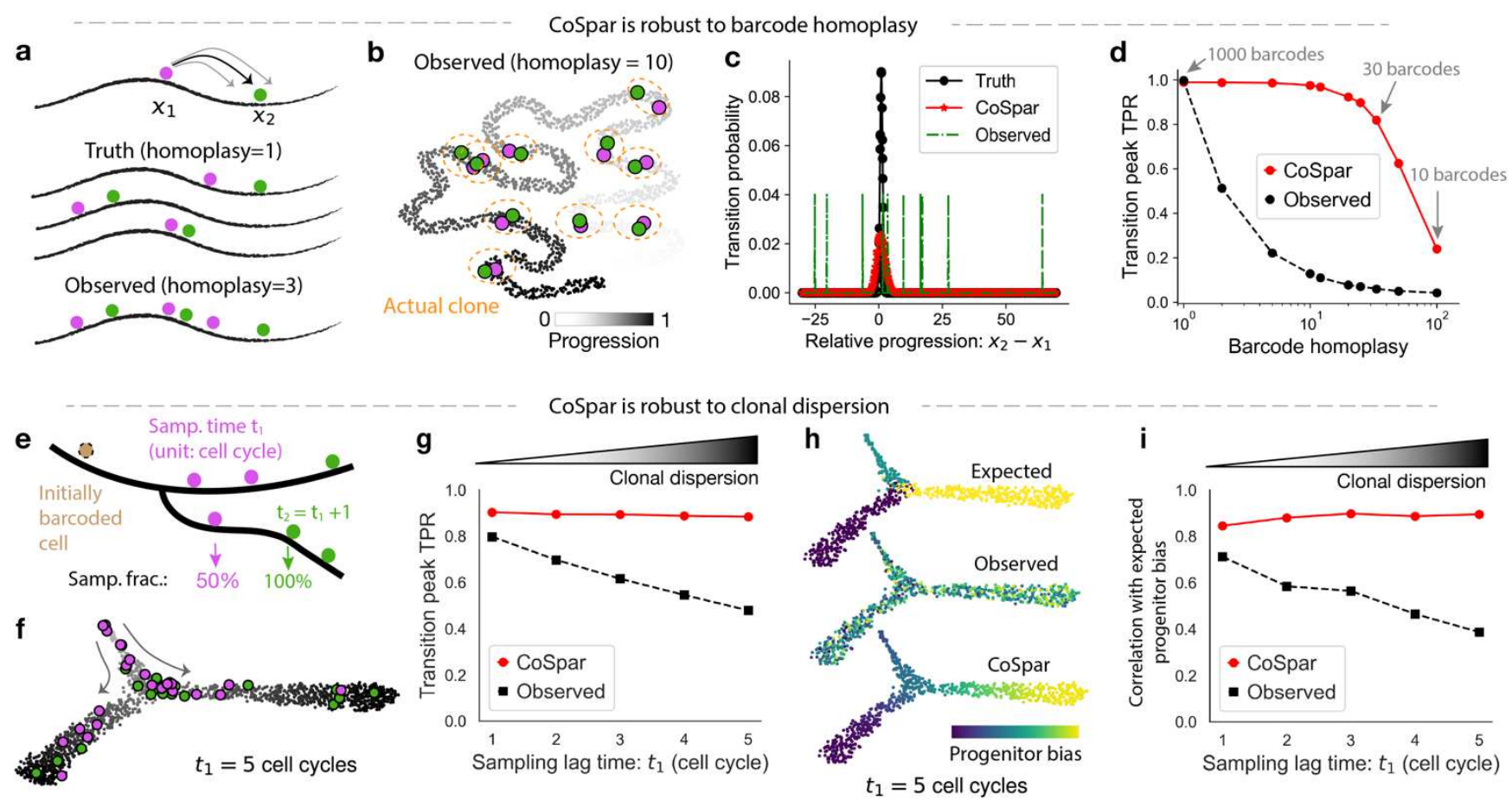

Fig. 3. Proof-of-concept with simulated data. a-d, Benchmarking transition map inference with barcode homoplasy errors. a, Schematics of a simplified simulated LT-scSeq experiment to evaluate the accuracy of CoSpar and its robustness to barcode homoplasy errors. Homoplasy is simulated by assigning multiple clones with the same barcode. b, UMAP embedding of simulated data. Cells labeled with one barcode are shown, with moderate homoplasy (10 clones / barcode). c, Distribution of true and inferred transition map matrix elements. Observed transitions are broadly distributed due to homoplasy errors, which associate progenitor cells and their progeny across different clones. CoSpar suppresses such transitions by enforcing sparsity and coherence. $\mathbf{d}$, CoSpar is robust to severe barcode homoplasy, as seen from the fraction of predicted transitions within 3 standard deviations of the true peak (TPR). e-i, Benchmarking transition map inference with clonal dispersion. e, Schematics of a second simulated LT-scSeq experiment including variable lag times between clonal labeling and observation. f, UMAP embedding of simulated data, with one example clone shown. The clone is first observed 5 cell divisions after initial labeling.

g, Quantitative evaluation of dynamic inference as a function of the sampling lag time. Growing lag time leads to higher clonal dispersion. Legend and transition peak TPR are defined as in $\mathbf{d}$. $\mathbf{h}$, Progenitor bias evaluated from the true and inferred transition maps with a simulated sampling lag time of five cell cycles. All clones are highly dispersed, providing no observed bias among early and late states; imposing sparsity enables recovering the true bias. i, Quantification of the correlation between true and inferred progenitor bias (shown in $\mathbf{h}$ ), over different sampling lag times. 

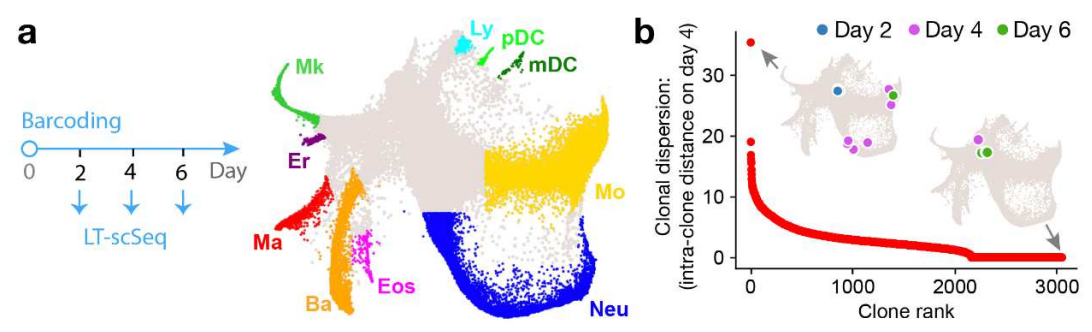

C Ground truth: Method from Weinreb et al., 2020

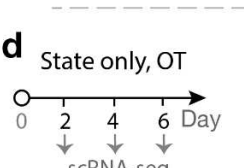

CoSpar predicts early fate bour
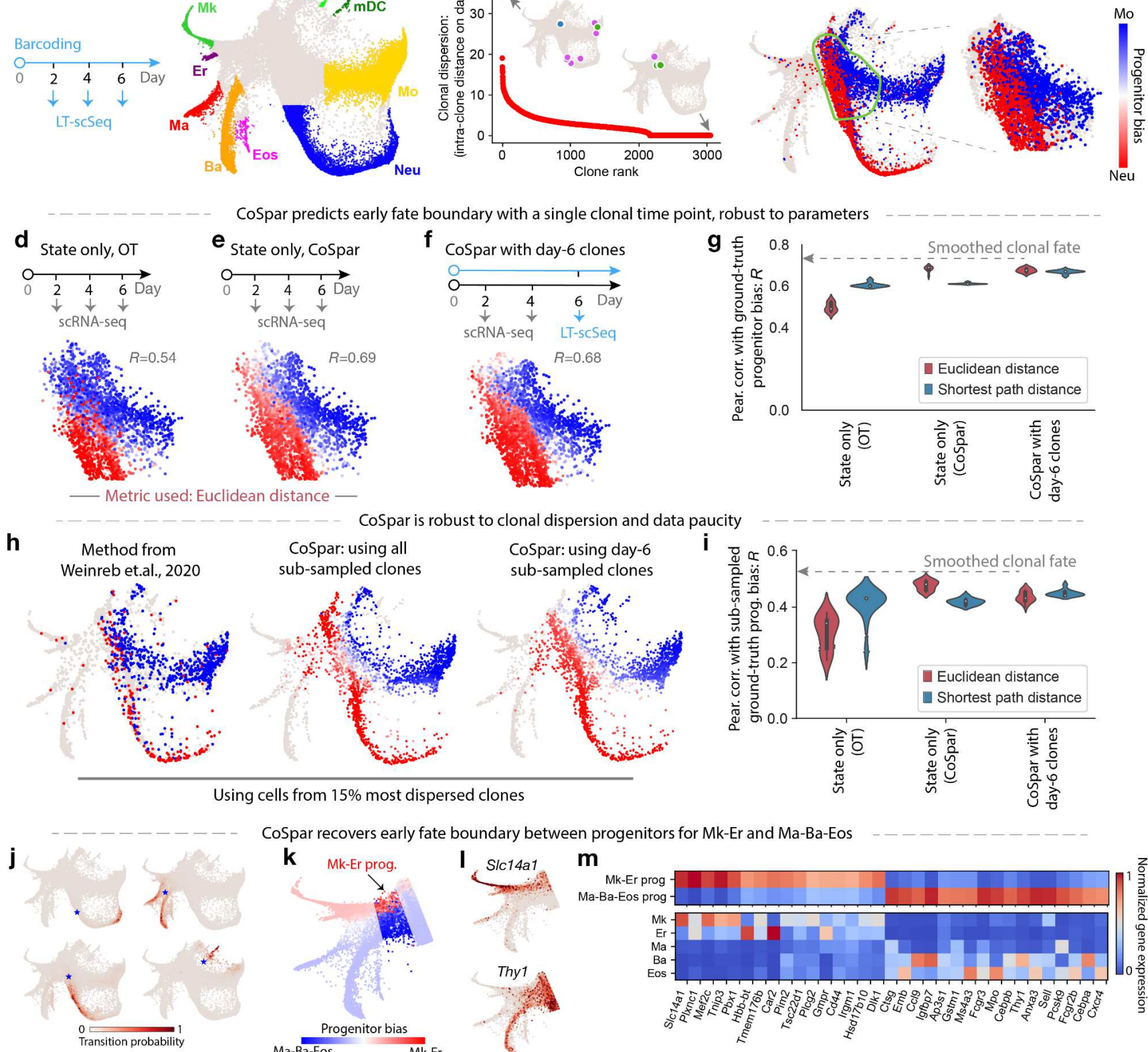

CoSpar is robust to clonal dispersion and data paucity
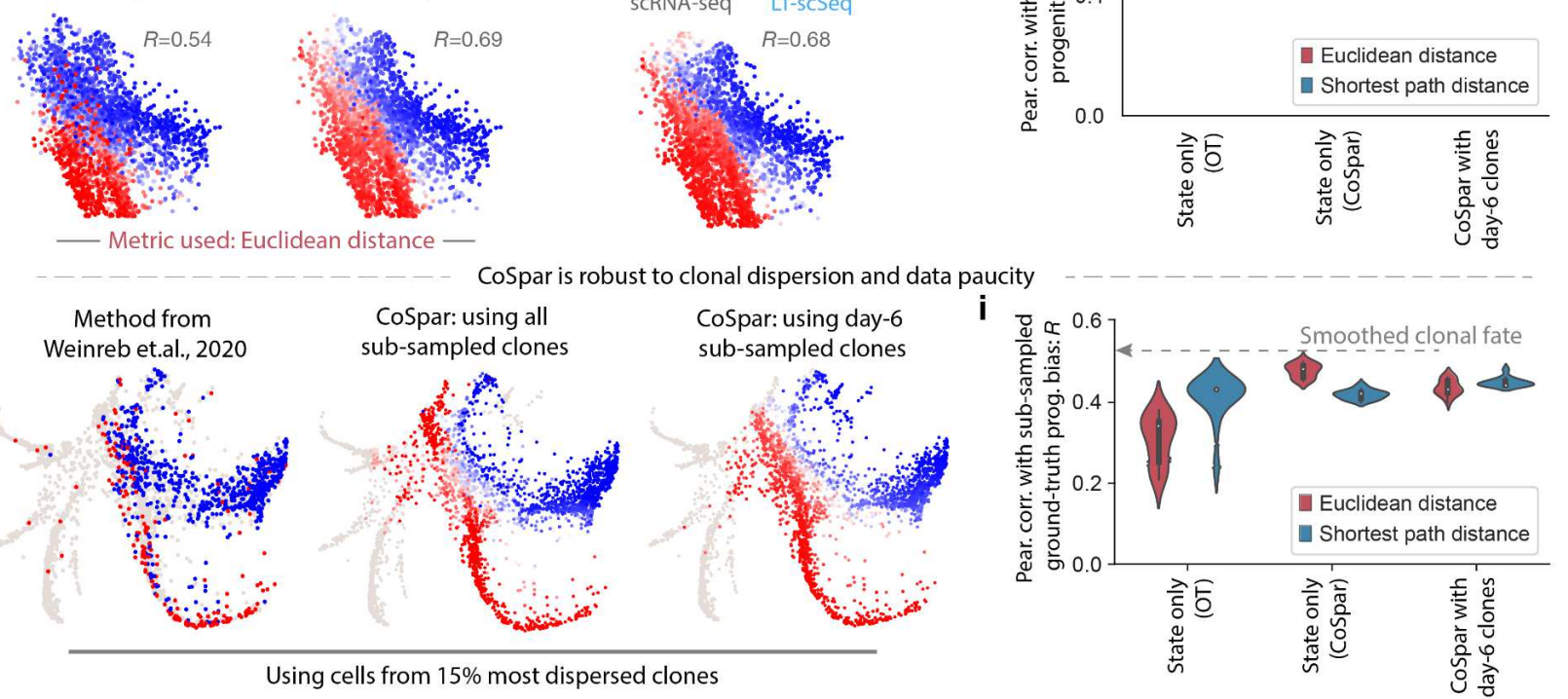

CoSpar recovers early fate boundary between progenitors for Mk-Er and Ma-Ba-Eos
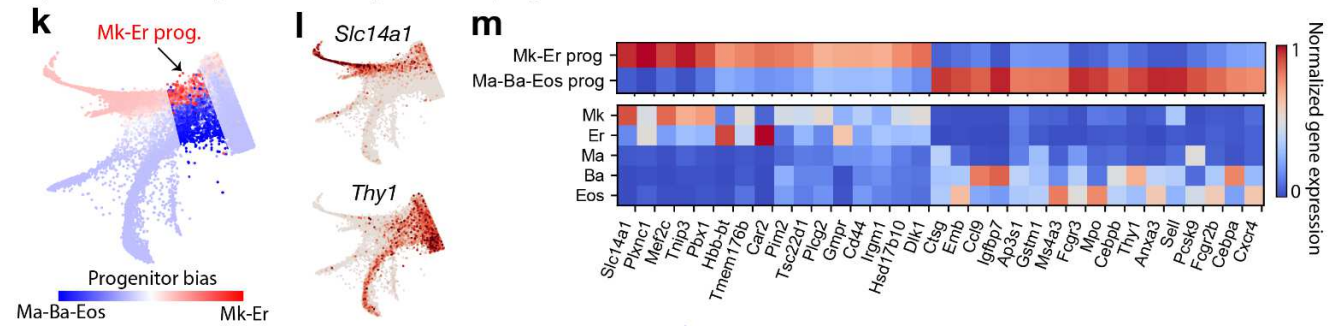

Fig. 4. Benchmarking CoSpar and prediction of progenitor bias in hematopoiesis. a, Experimental design and SPRING visualization of the hematopoiesis dataset from Weinreb et al. ${ }^{13}$. Early hematopoietic progenitors differentiate into megakaryocyte (Mk), erythrocyte (Er), mast cell (Ma), basophil (Ba), eosinophil (Eos), neutrophil (Neu), monocyte (Mo), lymphoid precursor (Ly), migratory (ccr7+) dendritic cells $(\mathrm{mDC})$, plasmacytoid DC $(\mathrm{pDC})$. b. Clones ranked by intra-clone dispersion (i.e., mean intra-clone graph distance) over the observed cell states after 4 days of differentiation. Two illustrative clones are shown. c, Bias towards Mo or Neu fate evaluated from all clonal data using the original method in Weinreb et $\mathrm{al}^{13}$. Bias among early progenitors (right panel) serves as ground truth for benchmarking. d,e, Baseline inference of progenitor bias using optimal transport (OT) or CoSpar, using only state information but no clonal data. 
f, CoSpar inference of progenitor bias using clonal data from a single-clonal time point. $\mathbf{g}$, Violin plot showing the distribution of fate prediction outcomes, quantified by the Pearson correlation of the inferred fate bias with the ground truth. The distribution reflects differences in parameters for the OT method (which is used to initialize CoSpar) and choice of distance metric used, showing that clonal data reduces sensitivity to parameter choices in data analysis. Dashed line shows the upper limit expected from cross-validation of benchmarking. h, Fate bias inferred using only the $15 \%$ most dispersed clones (ranked in panel b). i, Violin plots showing the distribution in inference performance with the down-sampled data (quantified as in $\mathbf{f}$ ) across parameter values. $\mathbf{j}$-m, Predicting the transcriptional identity of Gata1 ${ }^{+} \mathrm{Mk}-\mathrm{Er}$ and Ma-Ba-Eos progenitors using CoSpar. j, Representative values of the inferred transition map for 2-day transitions from 4 example cell states (indicated by ${ }^{*}$ ). $\mathbf{k}$, Heat map of predicted progenitor bias towards Mk-Er and Ma-Ba-Eos fates, overlaid on the state embedding. I, Expression of selected genes correlating strongly with predicted fate bias. $\mathbf{m}$, Expression heat map for selected genes differentially expressed between the Mk-Er and Ma-Ba-Eos progenitors. Full list of fate-associated genes is provided in Supplementary Table 1. 

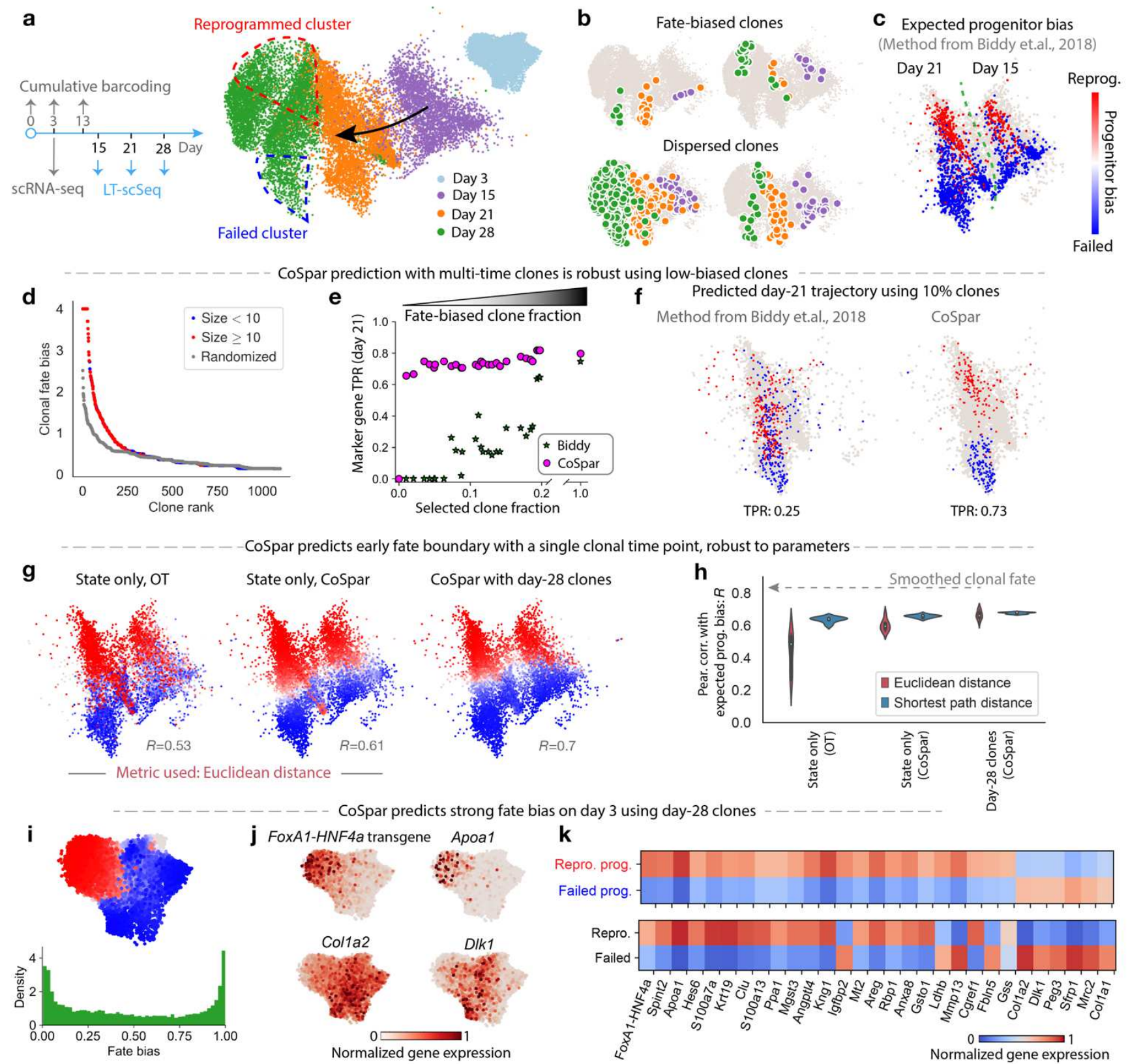

Fig. 5. Progenitor bias in fibroblast reprogramming. a, Experimental design and UMAP visualization of cell reprogramming from fibroblast cells to induced endoderm progenitors (iEP) by ectopic expression of a transgene FoxA1-HNF4a on day $0^{14}$. Schema shows time points for scRNA-seq only (grey arrows) and LT-scSeq (blue arrows). b, The UMAP visualization overlaid with examples of individual clones. Cells are colored by time point as in a. c, UMAP visualization of transcriptomes on days 15 and 21 of reprogramming, colored by progenitor bias towards successful or failed reprogramming fates, using cells in clones selectively filtered for strong fate bias as in the original study ${ }^{14}$. d-f, Benchmarking CoSpar using clones with weak fate bias. $\mathbf{d}$, Clones ranked by consistency in the fate outcomes of their constituent cells [fate bias defined as - $\log (p$-value), Fisher Exact test]. e, Accuracy in predicting the fate outcome of cells observed on day 21 using data from progressively fewer 
fate-biased clones. Predictions use the original method (Biddy et al. ${ }^{14}$ ) or CoSpar. Accuracy is assessed in the true positive rate (TPR) of identifying genes associated with fate outcomes previously reported in $\operatorname{Ref}^{14}$. f, UMAP visualization showing the cell states on day 21 predicted to undergo successful or failed reprogramming, when using the $10 \%$ clones with lowest fate bias. g-h, CoSpar predicts early progenitor bias with a single clonal time point, robust to parameters. g, Progenitor bias on days 15 and 21 predicted using only state information; or with end-point (day 28) clonal information only. $\mathbf{h}$, Violin plots as in Fig. $\mathbf{4 g}$ quantifying prediction accuracy over a range of parameters, showing consistent improvement by imposing coherence, sparsity, and enforcing clonal relationships. i-k, Predicting early fate determination within 3 days of transgene expression. i, Predicted progenitor bias of cells on day 3. j, Expression on day-3 states of selected genes predicted to correlate with successful or failed reprogramming. $\mathbf{k}$, Expression of additional genes differentially expressed on day 3 between cells predicted to succeed or to fail reprogramming. See the full list at Supplementary Table 2.
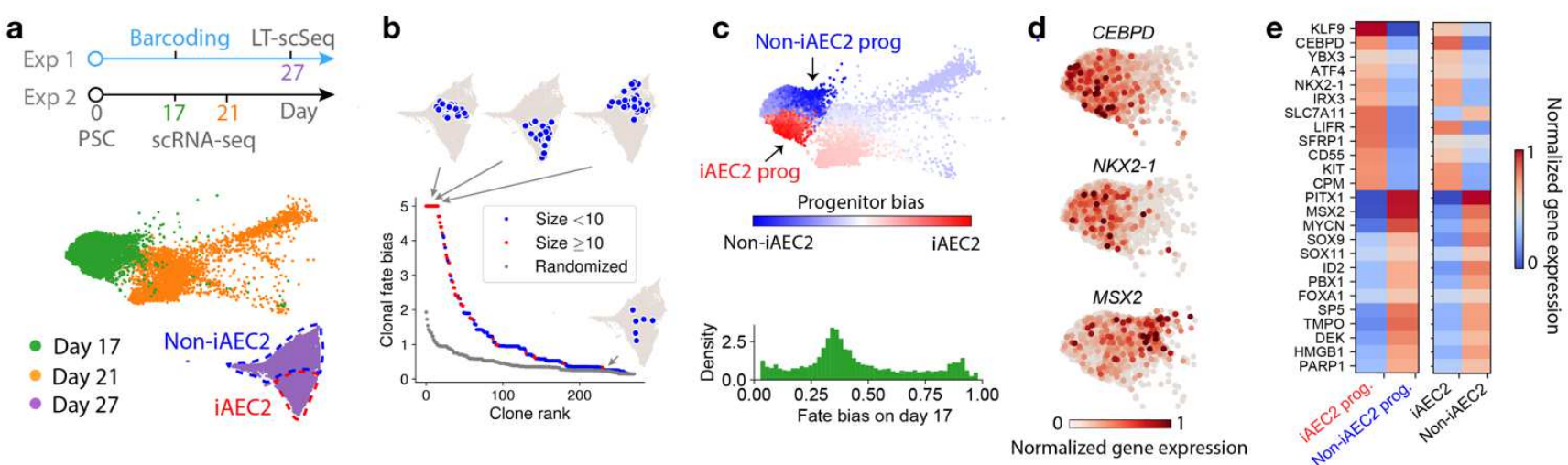

Fig. 6. Progenitor bias during hPSCs differentiation into endodermal lineages. a, Experimental design and UMAP visualization for differentiating human pluripotent stem cells (hPSC) into induced alveolar epithelium (iAEC2) lung cells and other endodermal cell types. b. Clones ranked by fate bias towards iAEC2 fate (bias defined as in Fig. $5 \mathrm{~d}$ ), with representative biased (top) and dispersed (bottom) clones shown. c, Predicted progenitor bias of cells towards iAEC2 fate on day 17 of differentiation, overlaid on the state embedding and shown as a histogram. d,e Expression on day-17 states of selected genes predicted to correlate with iAEC2 and non-iAEC2 fates. In e, expression is shown alongside the corresponding expression in mature cells on day 27.

\section{References}

1. Woodworth, M. B., Girskis, K. M. \& Walsh, C. A. Building a lineage from single cells: genetic techniques for cell lineage tracking. Nat. Rev. Genet. 18, 230-244 (2017). 
2. Wagner, D. E. \& Klein, A. M. Lineage tracing meets single-cell omics: opportunities and challenges. Nat. Rev. Genet. (2020) doi:10.1038/s41576-020-0223-2.

3. Kester, L. \& van Oudenaarden, A. Single-Cell Transcriptomics Meets Lineage Tracing. Cell Stem Cell 23, 166-179 (2018).

4. Haghverdi, L., Büttner, M., Wolf, F. A., Buettner, F. \& Theis, F. J. Diffusion pseudotime robustly reconstructs lineage branching. Nat. Methods 13, 845-848 (2016).

5. Trapnell, C. et al. The dynamics and regulators of cell fate decisions are revealed by pseudotemporal ordering of single cells. Nat. Biotechnol. 32, 381-386 (2014).

6. Bendall, S. C. et al. Single-cell trajectory detection uncovers progression and regulatory coordination in human B cell development. Cell 157, 714-725 (2014).

7. Schiebinger, G. et al. Optimal-Transport Analysis of Single-Cell Gene Expression Identifies Developmental Trajectories in Reprogramming. Cell 176, 928-943.e22 (2019).

8. Qiu, X. et al. Mapping Vector Field of Single Cells. 696724 (2019) doi:10.1101/696724.

9. Bergen, V., Lange, M., Peidli, S., Wolf, F. A. \& Theis, F. J. Generalizing RNA velocity to transient cell states through dynamical modeling. Nat. Biotechnol. (2020) doi:10.1038/s41587-020-0591-3.

10. La Manno, G. et al. RNA velocity of single cells. Nature 560, 494-498 (2018).

11. Tritschler, S. et al. Concepts and limitations for learning developmental trajectories from single cell genomics. Development 146, (2019). 
12. Weinreb, C., Wolock, S., Tusi, B. K., Socolovsky, M. \& Klein, A. M. Fundamental limits on dynamic inference from single-cell snapshots. Proc. Natl. Acad. Sci. U. S. A. 115, E2467-E2476 (2018).

13. Weinreb, C., Rodriguez-Fraticelli, A., Camargo, F. D. \& Klein, A. M. Lineage tracing on transcriptional landscapes links state to fate during differentiation. Science 367, (2020).

14. Biddy, B. A. et al. Single-cell mapping of lineage and identity in direct reprogramming. Nature 564, 219-224 (2018).

15. Rodriguez-Fraticelli, A. E. et al. Clonal analysis of lineage fate in native haematopoiesis. Nature 553, 212-216 (2018).

16. Rodriguez-Fraticelli, A. E. et al. Single-cell lineage tracing unveils a role for TCF15 in haematopoiesis. Nature (2020) doi:10.1038/s41586-020-2503-6.

17. Spanjaard, B. et al. Simultaneous lineage tracing and cell-type identification using CRISPR-Cas9-induced genetic scars. Nat. Biotechnol. 36, 469-473 (2018).

18. Alemany, A., Florescu, M., Baron, C. S., Peterson-Maduro, J. \& van Oudenaarden, A. Whole-organism clone tracing using single-cell sequencing. Nature 556, 108-112 (2018).

19. Chan, M. M. et al. Molecular recording of mammalian embryogenesis. Nature 570, 77-82 (2019).

20. Bowling, S. et al. An engineered CRISPR/Cas9 mouse line for simultaneous readout of lineage histories and gene expression profiles in single cells. Cell 797597 (2019) doi:10.1101/797597. 
21. Wagner, D. E. et al. Single-cell mapping of gene expression landscapes and lineage in the zebrafish embryo. Science 360, 981-987 (2018).

22. Lopez-Garcia, C., Klein, A. M., Simons, B. D. \& Winton, D. J. Intestinal stem cell replacement follows a pattern of neutral drift. Science 330, 822-825 (2010).

23. Hurley, K. et al. Reconstructed Single-Cell Fate Trajectories Define Lineage Plasticity Windows during Differentiation of Human PSC-Derived Distal Lung Progenitors. Cell Stem Cell (2020) doi:10.1016/j.stem.2019.12.009.

24. Yao, Z. et al. A Single-Cell Roadmap of Lineage Bifurcation in Human ESC Models of Embryonic Brain Development. Cell Stem Cell 20, 120-134 (2017).

25. Hormoz, S. et al. Inferring Cell-State Transition Dynamics from Lineage Trees and Endpoint Single-Cell Measurements. Cell Syst 3, 419-433.e8 (2016).

26. Tibshirani, R. Regression Shrinkage and Selection via the Lasso. J. R. Stat. Soc. Series B Stat. Methodol. 58, 267-288 (1996).

27. Tibshirani, R., Saunders, M., Rosset, S., Zhu, J. \& Knight, K. Sparsity and smoothness via the fused lasso. J. R. Stat. Soc. Series B Stat. Methodol. 67, 91-108 (2005).

28. Yu, V. W. C. et al. Epigenetic Memory Underlies Cell-Autonomous Heterogeneous Behavior of Hematopoietic Stem Cells. Cell 167, 1310-1322.e17 (2016).

29. Weissman, T. A. \& Pan, Y. A. Brainbow: new resources and emerging biological applications for multicolor genetic labeling and analysis. Genetics 199, 293-306 (2015).

30. Orkin, S. H. \& Zon, L. I. Hematopoiesis: an evolving paradigm for stem cell biology. Cell 132, 631-644 (2008). 
31. Ferreira, R., Ohneda, K., Yamamoto, M. \& Philipsen, S. GATA1 function, a paradigm for transcription factors in hematopoiesis. Mol. Cell. Biol. 25, 1215-1227 (2005).

32. Lu, Y.-C. et al. The Molecular Signature of Megakaryocyte-Erythroid Progenitors Reveals a Role for the Cell Cycle in Fate Specification. Cell Rep. 25, 2083-2093.e4 (2018).

33. Arinobu, Y. et al. Developmental checkpoints of the basophil/mast cell lineages in adult murine hematopoiesis. Proc. Natl. Acad. Sci. U. S. A. 102, 18105-18110 (2005).

34. Jacob, A. et al. Differentiation of Human Pluripotent Stem Cells into Functional Lung Alveolar Epithelial Cells. Cell Stem Cell 21, 472-488.e10 (2017).

35. Rockich, B. E. et al. Sox9 plays multiple roles in the lung epithelium during branching morphogenesis. Proc. Natl. Acad. Sci. U. S. A. 110, E4456-64 (2013).

36. Perl, A.-K. T., Kist, R., Shan, Z., Scherer, G. \& Whitsett, J. A. Normal lung development and function after Sox9 inactivation in the respiratory epithelium. Genesis 41, 23-32 (2005).

37. Treutlein, B. et al. Reconstructing lineage hierarchies of the distal lung epithelium using single-cell RNA-seq. Nature 509, 371-375 (2014).

38. Frieda, K. L. et al. Synthetic recording and in situ readout of lineage information in single cells. Nature 541, 107-111 (2017).

39. Ludwig, L. S. et al. Lineage Tracing in Humans Enabled by Mitochondrial Mutations and Single-Cell Genomics. Cell 176, 1325-1339.e22 (2019).

40. Raj, B. et al. Simultaneous single-cell profiling of lineages and cell types in the vertebrate brain. Nat. Biotechnol. 36, 442-450 (2018). 
41. McKenna, A. et al. Whole-organism lineage tracing by combinatorial and cumulative genome editing. Science 353, aaf7907 (2016).

42. Nitzan, M., Karaiskos, N., Friedman, N. \& Rajewsky, N. Gene expression cartography. Nature (2019) doi:10.1038/s41586-019-1773-3.

43. Stuart, T. \& Satija, R. Integrative single-cell analysis. Nat. Rev. Genet. 20, 257-272 (2019).

44. Cleary, B. et al. Compressed sensing for imaging transcriptomics. bioArxiv 743039 (2020) doi:10.1101/743039.

45. Nitzan, M., Casadiego, J. \& Timme, M. Revealing physical interaction networks from statistics of collective dynamics. Sci Adv 3, e1600396 (2017).

46. Jaitin, D. A. et al. Dissecting Immune Circuits by Linking CRISPR-Pooled Screens with Single-Cell RNA-Seq. Cell 167, 1883-1896.e15 (2016).

47. Adamson, B. et al. A Multiplexed Single-Cell CRISPR Screening Platform Enables Systematic Dissection of the Unfolded Protein Response. Cell 167, 1867-1882.e21 (2016).

48. Aggarwal, C. C. Recommender Systems: The Textbook. (Springer, Cham, 2016). 


\section{Supplementary Files}

This is a list of supplementary files associated with this preprint. Click to download.

- CoSparMethodsv9amk.pdf

- SupplementaryTable1hematopoiesismarkergenes.pdf

- SupplementaryTable2reprogrammingmarkergenes.pdf

- SupplementaryTable3lungmarkergenes.pdf

- CoSparsupplemetaryinformationv2.pdf 